\title{
External Cervical Resorption: A Case Report and Brief Review of the Literature, and Treatment Algorithms
}

\author{
Mohammed Howait ${ }^{1}$, Marwa Shaker ${ }^{2}$, Haila Aljuhani ${ }^{3}$, Manar AlMohnna $^{4}$
}

\begin{abstract}
Aim: This report presents a case of external cervical resorption and illustrates the effects of a non-surgical approach in the amelioration of this condition and discusses the etiology, classifications, and treatment options.

Background: One of the most common root resorption forms is external cervical resorption, which initiates in the cervical area of the tooth and spreads out in the thickness of the dentin in an irregular way. This resorptive process may spread across the dentin leading to significant loss of tooth structure, with or without pulp involvement.

Case description: During a routine radiographic examination of a 25 -year-old female patient, external cervical resorption in a maxillary right second premolar was discovered. Cone-beam computed tomography (CBCT) confirmed the extension of the lesion into the pulp and the need for root canal treatment. The defect was sealed with bioceramic putty. One year CBCT follow-up demonstrated the cessation of the resorption site with no clinical symptoms.

Conclusion: $\mathrm{CBCT}$ examination and combining non-surgical root canal treatment with non-surgical repair using bioceramic putty was an effective treatment option.

Clinical significance: Treatment selection of external cervical resorption depends on many factors, including the location and severity of the resorptive defect and the remaining tooth structure. If the resorptive defect has extended to the pulp, the management involves root canal treatment and subsequent placement of a direct restoration to restore the resorptive lesion.
\end{abstract}

Keywords: Apical periodontitis, External cervical resorption, Periapical lesion, Root resorption.

The Journal of Contemporary Dental Practice (2021): 10.5005/jp-journals-10024-3013

\section{BACKGROUND}

Root resorption is a condition characterized by the loss of hard dental tissues. It is considered a desirable physiological process in primary teeth, whereas resorption of the permanent teeth can be a pathologic process leading to permeant tooth loss. ${ }^{1}$

Based on the location, root resorption can be classified into internal, when it starts within the pulp, or external when it starts from the periodontium into the outer surface of the tooth. The latter is further classified into external inflammatory resorption, external surface resorption, external cervical resorption, and external replacement resorption. ${ }^{2}$

External cervical resorption (ECR) is the aggressive pathological resorption that initiates at the cervical region below the epithelial attachment of the root. ${ }^{3}$ This resorptive process may spread across the dentin with or without pulp involvement, which leads to significant loss of tooth structure. ECR is usually asymptomatic due to the presence of the pericanalar resorption-resistant sheet (PRES), which surrounds the root canal and acts as a protective layer against resorption. ${ }^{4}$ ECR is usually detected during routine radiograph examination, which usually appears as an asymmetrical radiolucent lesion with ill-defined borders in the cervical area of the tooth. In advanced cases, ECR may perforate the PRES causing individuals to experience pulpal symptoms. ${ }^{1,4,5}$

\section{Etiology}

The etiology of ECR remains unknown. Several predisposing factors have been proposed that could damage the cervical region of the tooth and consequently initiate ECR. These factors include

\begin{abstract}
1,2Department of Endodontics, King Abdul Aziz University, Jeddah, Kingdom of Saudi Arabia

${ }^{3}$ Private Practice, Jeddah, Kingdom of Saudi Arabia

${ }^{4}$ Ministry of Health, Taif, Kingdom of Saudi Arabia

Corresponding Author: Mohammed Howait, Department of Endodontics, Faculty of Dentistry, King Abdul Aziz University, Jeddah, Kingdom of Saudi Arabia, Phone: +966 2 6404002, e-mail: mhowait@ kau.edu.sa

How to cite this article: Howait M, Shaker M, Aljuhani $\mathrm{H}$, et al. External Cervical Resorption: A Case Report and Brief Review of the Literature, and Treatment Algorithms. J Contemp Dent Pract 2021;22(3):298-303.
\end{abstract}

Source of support: Nil

Conflict of interest: None

orthodontic treatment, dental trauma, dentoalveolar surgery, internal bleaching, and periodontal therapy. ${ }^{6}$

\section{Classification}

Heithersay established a clinical classification of external cervical resorption. Class 1 presents with a minor defect close to the cervical region with superficial penetration into dentine; Class 2 when the invasive resorptive lesion extends to the coronal pulp but with little or no extension into the radicular dentin; Class 3 when resorption presents with much deeper penetration into at least one-third of the root dentine; and Class 4 presents with an extensive invasive resorptive lesion that has penetrated more than one-third of the root. ${ }^{6}$ 


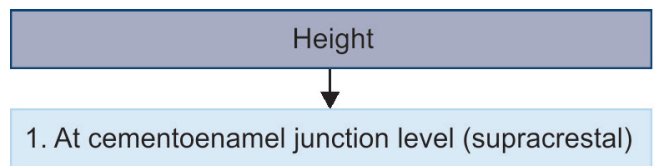

2. Extends into coronal third of root (subcrestal)

3. Extends into mid-third of the root

4. Extends into apical-third of the root

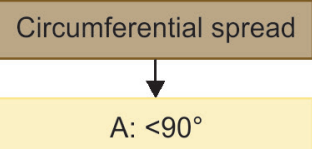

B: $>90^{\circ}$ to $\leq 180^{\circ}$

C: $>180^{\circ}$ to $\leq 270^{\circ}$

$\mathrm{D}:<270^{\circ}$

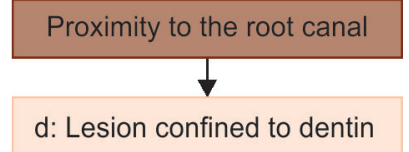

p: Probable pulpal involvement

Fig. 1: A three-dimensional classification for ECR (Reproduced from Patel et al., author permission was acquired) ${ }^{8}$

Heithersay's classification of ECR is based on two-dimensional radiographs, which can result in underestimating the actual extension of the resorptive lesion. ${ }^{7}$ Therefore, Patel et al. introduced a new three-dimensional classification based on the height of the lesion, circumferential extension, and proximity to the root canal using both periapical radiograph and cone-beam computed tomography $(\mathrm{CBCT})$ as shown in Figure $1 .^{8}$

\section{Treatment}

Treatment selection depends on many factors, including the location and severity of the resorptive defect and the remaining tooth structure. Treatment options are summarized as the following:

\section{External Repair of the Resorptive Lesion}

Small defects, such as in Patel class 1Ad, 2Ad, and 2Bd, could be restored with direct restorations while cases with pulpal involvement, for example, in Patel class $1 \mathrm{Ap}, 2 \mathrm{Ap}$, and $2 \mathrm{Bp}$, endodontics root canal treatment may be indicated. ${ }^{7}$ This treatment option includes a reflection of a flap to get adequate access to the ECR lesion to curette the granulomatous tissues using a sharp excavator. ${ }^{9}$ Trichloroacetic acid (TCA) is then applied to the excavated cavity to encourage coagulation necrosis to the granulomatous tissue. ${ }^{10}$ Alternatively, sodium hypochlorite can be used to dissolve the granulation tissues. ${ }^{11}$ Composite resin or glass ionomer should then be used to restore the excavated cavity. ${ }^{12}$ If endodontic treatment is indicated, patency of the canal should be preserved by placing a gutta-percha point inside the canal(s) and then, the flap repositioned and sutured. Root canal treatment must be completed after the restoration of the ECR lesion. ${ }^{13}$

\section{Internal Repair and Root Canal Treatment}

This line of treatment is suggested when the lesion is inaccessible using the external (surgical) approach, such as in Patel class $2 \mathrm{Cp}$, 2Dp, 3Cp, and 3Dp. ${ }^{7}$ Management involves root canal treatment and subsequent placement of a direct restoration to restore the resorptive lesion. The access cavity has to be extended to include the ECR lesion. ${ }^{11}$

\section{Intentional Replantation}

In teeth where the ECR is located interproximally or at the middle third of roots near to the apical region, for example, Patel class $3 \mathrm{Ad}$ and 3Bd, intentional replantation should be considered. ${ }^{7}$

Intentional replantation is started by atraumatic extraction of the tooth followed by debridement of the defect then placement of a restoration, followed by replanting the tooth within 15 minutes. ${ }^{11}$
The extracted tooth should be handled from the crown and kept hydrated in saline ${ }^{14}$ or Hank's balanced salt solution to avoid desiccation. ${ }^{15}$ After the tooth is replanted, it should be stabilized with a flexible splint for up to two weeks. ${ }^{16}$ The patient is then recalled two weeks after the procedure to remove the splint and annual follow-ups, thereafter. ${ }^{17}$ Critical factors for successful intentional replantation are atraumatic extraction, ${ }^{18}$ decreasing extraoral dry time to less than 15 minutes, ${ }^{19}$, and preservation of cementum and periodontal tissue. ${ }^{14}$

\section{Periodic Review}

Untreatable teeth may be followed up periodically (preferably annually) to document the progression of the resorptive process, such as in Patel class 2-4Dd and 2-4Dp. ${ }^{7}$ This periodic follow up should include evaluation of periodontal health, the progression of the resorptive lesion, any color changes to the crown, pulp status, and radiographic assessment. ${ }^{11}$

\section{Extraction}

Indicated when ECR is unapproachable to any treatment option, or when the tooth is not restorable because the defect is too extensive. ${ }^{11}$

\section{Prognosis}

Small-sized resorption lesions and lesions located in an accessible region have the most favorable prognosis. ${ }^{7}$

\section{Case Report}

A healthy 26-year-old female patient visited the general dentist for a regular check-up. Her medical and family histories were noncontributory. Clinical examination showed an overall fair oral hygiene with a healthy periodontal condition and multiple carious teeth. The patient did not report a history of trauma or tooth bleaching, but she had non-extraction orthodontic treatment to correct her overjet and crowding that lasted 24 months.

Routine dental radiographs that included full mouth preapical and two bitewing radiographs were taken which revealed a poorly defined radiolucent lesion in the cervical area of the maxillary right second premolar (Fig. 2A and B). The patient was then referred to an endodontist. Tooth \#15 was sound with no restoration or caries detected. Also, there was no evidence of a sinus tract or swelling in the region. Pulp sensitivity tests including cold and electrical stimulus revealed a positive response. The tooth was not tender to palpation, biting, or percussion. No mobility was found. To exclude the presence of root caries, an explorer probe was introduced within 

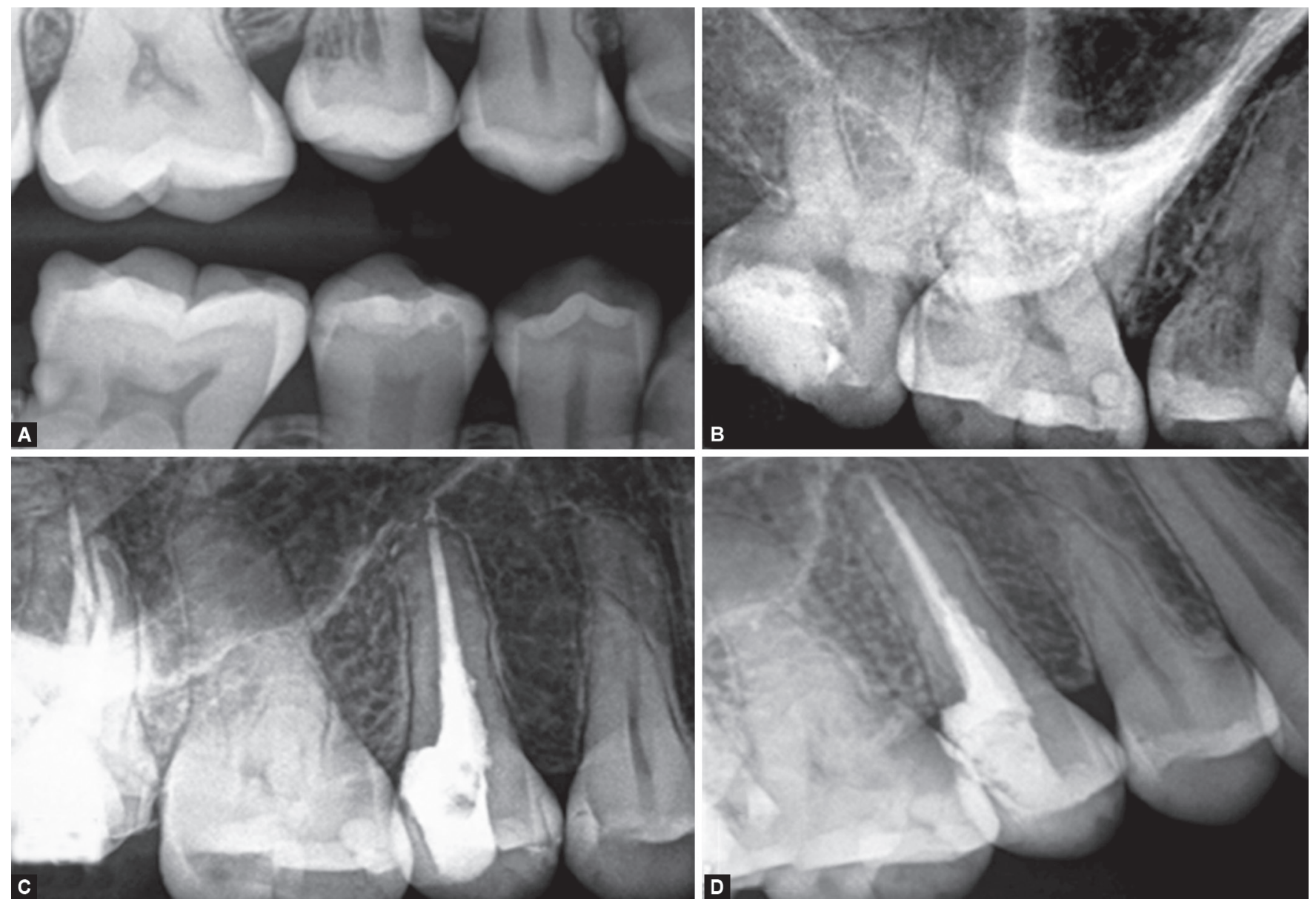

Figs 2A to D: Radiographic images of tooth 15: (A) Preoperative bitewing radiograph; (B) Preoperative periapical radiograph; (C) Postoperative radiograph; and (D) One-year recall radiograph

the cavity to check for any hardened and roughened surface. The resorptive defect was classified as Heithersay class III.

A CBCT scan was taken using CS 9300 CBCT scanner (Carestream Health, Inc., Rochester, NY, USA) to determine the nature and severity of the resorptive defect. The scan was viewed using the CS 3D imaging software (Kodak Dental Imaging Software, Carestream Dental, Atlanta, GA, USA), which revealed an advanced resorptive defect in tooth \#15. The axial and sagittal images revealed cervical root resorption, which extended into more than one-third of the root. The circumferential spreading of the lesion exceeded 180 degrees (Fig. 3A). Furthermore, the extension of the lesion into the pulp chamber could also be observed clearly in the CBCT images. Thus, the case was classified as Patel class 3CP.

All treatment options, including extraction and internal repair with root canal treatment, were discussed with the patient. Internal repair with root canal treatment was planned, taking into consideration the patient's desire to save the tooth. Written consent from the patient was taken.

After administering local anesthesia (lidocaine $2 \% \mathrm{HCl}$ injection, USP, Huons Co., Ltd, Seongnam, Korea), root canal treatment was performed under proper rubber dam isolation. The access cavity was prepared and extended to include the resorptive defect. The canal was then cleaned and shaped by Reciproc ${ }^{\circledR}$ blue size $R 40$ rotary file (VDW GmbH, Munich, Germany), irrigated with $5.25 \% \mathrm{NaOCl}$ and dried with paper points. Using an operating microscope, the resorptive defect was cleaned using ultrasonic tips (Spartan, Fenton, MI, USA), irrigated profusely with $2.5 \%$ sodium hypochlorite, dried and filled with bioceramic putty (EndoSequence BC RMMFast Set Putty, Brasseler USA, Savannah, GA, USA). The canal was restored and filled with a single cone of Reciproc ${ }^{\circledR}$ Gutta Percha R40 (VDW GmbH, Munich, Germany). The composite restoration was used to restore the tooth.

The patient was seen one month after the treatment. The tooth was symptom-free, there were no changes in the degree of mobility, or pain in response to palpation, biting, or percussion. Radiographical examination showed favorable healing (Fig. 2C).

The patient was seen after 12 months. Tooth \#15 was still asymptomatic with no evidence of progression of ECR on radiographic images (Fig. 2D) and $C B C T$ images were obtained (Fig. 3B) showing healthy bone and periodontal structures. The patient was instructed to maintain good oral hygiene and her annual follow-up visits.

\section{Discussion}

External cervical resorption is defined as inflammatory resorption of dental hard tissues (dentin and cementum) by inflammatory clastic cells (osteoclasts). ${ }^{1}$ As discussed earlier, several predisposing factors might be related to the etiology of ECR. In the present case, the patient had undergone orthodontic treatment in the past that 

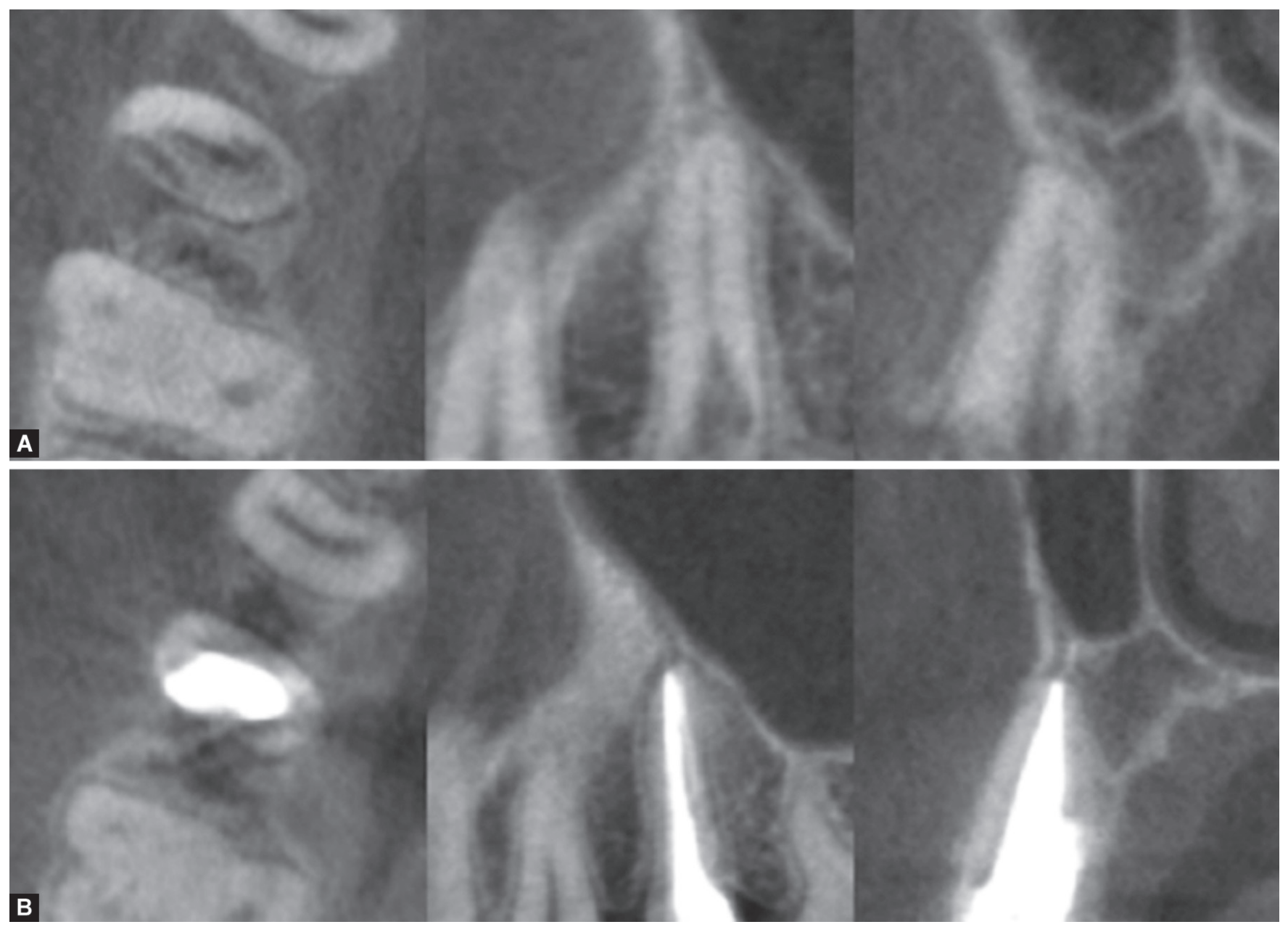

Figs $3 \mathrm{~A}$ and B: (A) The axial section shows the circumferential extension of the resorptive lesion, which surrounds more than $180^{\circ}$ of root dentin with pupal involvement and the sagittal/coronal sections show the subcrestal extension of the resorptive lesion into the coronal third of the root; (B) One-year recall CBCT images

may have predisposed to ECR. According to Heithersay, orthodontic treatment was found to be the most common factor associated with ECR. ${ }^{6}$ During orthodontic treatment, the application of excessive forces at the cervical area of the tooth may result in necrosis of the cementum and adjacent tissue leading to exposure of root dentin. As a result, this might stimulate the differentiation of mononuclear precursor cells into odontoclasts, resulting in resorption of the exposed root dentin. Studies by Heithersay ${ }^{6}$ and Mavridou et al. ${ }^{20}$ found that orthodontic treatment followed by a history of trauma were the two most frequently observed local predisposing factors to ECR. Orthodontics was the most common risk factor found in 45.7 and $21.2 \%$ of patients with ECR. Proper diagnosis is essential for every case, and it becomes more critical in challenging cases. Therefore, in addition to the clinical and periapical examination, it is recommended to use CBCT in certain cases when the diagnosis is debatable, and during treatment planning for ECR. ${ }^{7}$ This case report illustrates the importance of using CBCT imaging as a noninvasive diagnostic technique to give the clinician precise details about the resorption defect location, size, true extension of the lesion, and its proximity to the root canal ${ }^{21}$ to make a confirmatory diagnosis and better treatment outcome of ECR. CBCT allows ECR to be viewed in different planes without any geometric distortion or superimposition of overlying structures. ${ }^{22}$ Several studies have confirmed that CBCT is more accurate than periapical radiographs in external resorption diagnosis and assessment. ${ }^{23-25}$

The finding of cervical root resorption with pulpal involvement in CBCT confirmed that the resorptive process has extended below the coronal third of the root. It has also perforated the pericanalar resorption-resistant sheet (PRES). The main goal of treating this resorptive defect is to remove the resorptive tissue and cut the blood supply to the existing odontoclasts to inactivate the resorptive process. The defect must be completely debrided and sealed to prevent revascularization and further clastic action. ${ }^{26}$ Selecting the best treatment option for ECR is depends mainly on the extension of the defect. The root resorption, in this case, was extensive and with the involvement of the root canal (Patel class $3(P)$; thus, the chosen treatment was internal repair with endodontic root canal treatment. ${ }^{7}$ The favorable outcome in this case, confirms that the internal treatment approach is a suitable treatment option in cases of advanced ECR.

This highlights the importance of Patel classification to accurately classify ECR lesions using CBCT imaging. When the lesion is accurately diagnosed, it will have a positive impact on the treatment plan and outcome, which shows the impact of utilizing 
CBCT in ECR diagnosis and assessment of the relationship between ECR and potential local predisposing factors.

The ultimate treatment goal of a tooth with any resorption is to arrest the active resorption process, restore the defect site, and maintain the function of the tooth. To restore a resorptive defect, several materials have been used. Bioceramic materials, such as mineral trioxide aggregates (MTA) (Dentsply-Tulsa Dental, Johnson City, TN, USA) are considered the material of choice in terms of biocompatibility and tissue response. The ability of MTA to induce reparative dentinogenesis or dentin bridge formation has been consistently reported in several studies. ${ }^{27,28}$ Compared with calcium hydroxide, MTA induces reparative dentin formation at a higher rate and better structural integrity. ${ }^{29,30}$ However, due to its long setting time, it was not used in this case. Alternatively, bioceramic putty (EndoSequence BC RMM-Fast Set Putty, Brasseler USA, Savannah, GA, USA) was used. It is a highly biocompatible bioceramic material with superior handling properties because it comes in premixed syringe delivery. Moreover, it has a faster setting time (approximately $20 \mathrm{~min}$ ), which overcomes the long setting time of MTA. ${ }^{31}$ Additionally, even though this case report showed a favorable outcome, further studies are recommended to support the use of bioceramic putty to restore the defect of external cervical resorption. To overcome the low prevalence of ECR which might limit the sample size for a prospective cohort study, we recommend future multicenter cohort studies. Also, long-term studies with longer follow-up periods are needed to determine and evaluate the influence of the classification of ECR and the choice of repair material on the treatment outcome.

\section{Conclusion}

Successful management of ECR depends mainly on the early detection of the lesion. CBCT appears to be an auspicious diagnostic tool for confirming the presence, detecting the true nature, and evaluating the management of ECR. Bioceramic putty is a biocompatible repair material with a fast setting time. It showed good results when treating ECR with an internal repair.

\section{Clinical Significance}

Treatment selection depends on many factors, including the location and severity of the resorptive defect and the remaining tooth structure. If the resorptive defect has extended to the pulp, the management involves root canal treatment and subsequent placement of a direct restoration to restore the resorptive lesion.

\section{References}

1. Patel $S$, Kanagasingam S, Pitt Ford T. External cervical resorption: a review. J Endod 2009;35(5):616-625. DOI: 10.1016/j.joen.2009.01.015.

2. Patel S, Ford TP. Is the resorption external or internal? Dent Update 2007;34(4):218-220, 222, 224-226, 229. DOI: 10.12968/ denu.2007.34.4.218.

3. Patel J, Beddis HP. How to assess and manage external cervical resorption. Br Dent J 2019;227(8):695-701. DOI: 10.1038/s41415-0190781-x.

4. Mavridou AM, Hauben $E$, Wevers $M$, et al. Understanding external cervical resorption in vital teeth. J Endod 2016;42(12):1737-1751. DOI: 10.1016/j.joen.2016.06.007.

5. Liang H, Burkes EJ, Frederiksen NL. Multiple idiopathic cervical root resorption: systematic review and report of four cases. Dentomaxillofac Radiol 2003;32(3):150-155. DOI: 10.1259/dmfr/12925020.
6. Heithersay GS. Invasive cervical resorption: an analysis of potential predisposing factors. Quintessence Int 1999;30(2):83-95. Retrieved from http://www.quintpub.com/journals/qi/.

7. European Society of Endodontology developed by; Patel S, Lambrechts P, et al. European Society of Endodontology position statement: external cervical resorption. Int Endod J 2018;51(12):1323-1326. DOI: 10.1111/iej.13008.

8. Patel S, Foschi F, Mannocci F, et al. External cervical resorption: a three-dimensional classification. Int Endod J 2018;51(2):206-214. DOI: $10.1111 /$ iej.12824.

9. Frank AL, Torabinejad M. Diagnosis and treatment of extracanal invasive resorption. J Endod 1998;24(7):500-504. DOI: 10.1016/S00992399(98)80056-3.

10. Heithersay GS. Treatment of invasive cervical resorption: an analysis of results using topical application of trichloracetic acid, curettage, and restoration. Quintessence Int 1999;30(2):96-110. Retrieved from http://www.quintpub.com/journals/qi/.

11. Patel S, Foschi F, Condon R, et al. External cervical resorption: part 2-management. Int Endod J 2018;51(11):1224-1238. DOI: 10.1111/ iej.12946.

12. Heithersay GS. Management of tooth resorption. Aust Dent J 2007;52(1 Suppl):S105-S121. DOI: 10.1111/j.1834-7819.2007.tb00519.x.

13. Patel S, Durak C, Ricucci D. Chapter 16, Root resorption. In: Berman L, Hargreaves K, eds. Cohen's Pathways of the Pulp, 11th edition. St Louis, MO: Elsevier; 2016. pp. 660-683. ISBN: 9780323185875. Retrieved from https://www.elsevier.com/books/cohens-pathwaysof-the-pulp-expert-consult/berman/978-0-323-09635-5.

14. Becker BD. Intentional replantation techniques: a critical review. J Endod 2018;44(1):14-21. DOI: 10.1016/j.joen.2017.08.002.

15. Kratchman S. Intentional replantation. Dent Clin North Am 1997:41(3):603-617. Retrieved from https://www.dental.theclinics.com.

16. Andersson L, Andreasen JO, Day P, et al. Guidelines for the management of traumatic dental injuries: 2 . Avulsion of permanent teeth. Pediatr Dent 2016;38(6):369-376. DOI: 10.1111/j.16009657.2012.01125.x

17. Cho SY, Lee Y, Shin SJ, et al. Retention and healing outcomes after intentional replantation. J Endod 2016;42(6):909-915. DOI: 10.1016/j. joen.2016.03.006.

18. Choi YH, Bae JH, Kim YK, et al. Clinical outcome of intentional replantation with preoperative orthodontic extrusion: a retrospective study. Int Endod J 2014;47(12):1168-1176. DOI: 10.1111/iej.12268.

19. Torabinejad M, Dinsbach NA, Turman M, et al. Survival of intentionally replanted teeth and implant-supported single crowns: a systematic review. J Endod 2015;41(7):992-998. DOI: 10.1016/j. joen.2015.01.004.

20. Mavridou AM, Bergmans L, Barendregt $D$, et al. Descriptive analysis of factors associated with external cervical resorption. J Endod 2017;43(10):1602-1610. DOI: 10.1016/j.joen.2017.05.026.

21. Patel K, Mannocci F, Patel S. The assessment and management of external cervical resorption with periapical radiographs and cone-beam computed tomography: a clinical study. J Endod 2016;42(10):1435-1440. DOI: 10.1016/j.joen.2016.06.014.

22. Patel S, Durack C, Abella F, et al. Cone beam computed tomography in endodontics—a review. Int Endod J 2015;48(1):3-15. DOI: 10.1111/ iej.12270.

23. Bernardes RA, de Paulo RS, Pereira LO, et al. Comparative study of cone beam computed tomography and intraoral periapical radiographs in diagnosis of lingual-simulated external root resorptions. Dent Traumatol 2012;28(4):268-272. DOI: 10.1111/j.16009657.2011.01113.x.

24. Ren H, Chen J, Deng F, et al. Comparison of cone-beam computed tomography and periapical radiography for detecting simulated apical root resorption. Angle Orthod 2013;83(2):189-195. DOI: 10.2319/050512-372.1.

25. Vaz de Souza D, Schirru E, Mannocci F, et al. External cervical resorption: a comparison of the diagnostic efficacy using 2 different cone-beam computed tomographic units and periapical radiographs. J Endod 2017;43(1):121-125. DOI: 10.1016/j.joen.2016.09.008. 
26. Heithersay GS. Invasive cervical resorption. Endodontic Topics. 2004;7(1):73-92. DOI: 10.1111/j.1601-1546.2004.00060.x.

27. Yamamura T. Differentiation of pulpal cells and inductive influences of various matrices with reference to pulpal wound healing. J Dent Res 1985;64 Spec No:530-540. DOI: 10.1177/002203458506400406.

28. Kuratate $M$, Yoshiba K, Shigetani Y, et al. Immunohistochemical analysis of nestin, osteopontin, and proliferating cells in the reparative process of exposed dental pulp capped with mineral trioxide aggregate. J Endod 2008;34(8):970-974. DOI: 10.1016/j.joen.2008.03.021.
29. Ford TR, Torabinejad $M$, Abedi HR, et al. Using mineral trioxide aggregate as a pulp-capping material. J Am Dent Assoc 1996;127(10):1491-1494. DOI: 10.14219/jada.archive.1996.0058.

30. Faraco IM Jr., Holland R. Response of the pulp of dogs to capping with mineral trioxide aggregate or a calcium hydroxide cement. Dent Traumatol 2001;17(4):163-166. DOI: 10.1034/j.1600-9657.2001.170405.x.

31. Gilberto Debelian MT. The use of premixed bioceramic materials in endodontics. Giornale Italiano di Endodonzia 2016;30(2):70-80. DOI: 10.1016/j.gien.2016.09.001. 